Final Report to DOE CMSN — 5/1/2007 - 4/30/2011

\title{
Predictive Capability for Strongly Correlated Systems:
}

Mott Transition in MnO, Multielectron Magnetic Moments, and Dynamic Effects in Correlated Materials

Cyrus Umrigar

Cornell University

Postdoc Supported. W. A. Al-Saidi (Cornell) 


\section{Correlated Wavefunctions}

This project was one of several projects worked on by the multi-institutional CMCN team headed by Warren Pickett and Richard Scalettar at the University of California, Davis. At Cornell, Umrigar, Toulouse, Al-Saidi, Changlani, Kinder and Chan contributed to the project. We also collaborated with the group of Professor Harold Baranger at Duke University and with a student Paul Zimmerman at Stanford (currently a postdoc at Berkeley).

Diffusion Monte Carlo methods can give highly accurate results for correlated systems, provided that well optimized trial wave functions with accurate nodal surfaces are employed. The Cornell team developed powerful methods for optimizing all the parameters within a multi-determinant Slater-Jastrow form of the wave function [1]. These include the Jastrow parameters within a flexible electron-electron-nucleus form of the Jastrow function, the parameters multiplying the configuration state functions, the orbital parameters and the basis exponents. The method optimizes a linear combination of the energy and the variance of the local energy. The optimal parameters are found iteratively by diagonalizing the Hamiltonian matrix in the space spanned by the wave function and its first-order derivatives, making use of a strong zero-variance principle. It is highly robust, has become the method of choice for correlated wave function optimization and has been adopted by other QMC groups.

This optimization method was used on the first-row atoms and homonuclear diatomic molecules, demonstrating that molecular well depths can be obtained with near chemical accuracy quite systematically at the diffusion Monte Carlo level for these systems [1]. In addition the complete ground-state potential energy curve of the $\mathrm{C}_{2}$ molecule up to the dissociation limit was obtained, and, size consistency and broken spin-symmetry issues in quantum Monte Carlo calculations were studied [1].

The method was used with a eight-electrons-in-eight-orbitals complete active space CAS $(8,8)$ wave function to study the relative energies of the monocyclic and bicyclic forms of m-benzyne [2]. The DMC calculations show that the monocyclic structure is lower in energy than the bicyclic structure by $1.92 \mathrm{kcal} /$ mole, which is in excellent agreement with the best coupled cluster results $(\mathrm{CCSD}(\mathrm{T}))$ and in disagreement with the CCSD results.

QMC methods have for the most part been used only for ground states of a given symmetry. We developed a method for calculating low-lying excited states as well and tested it on the ground and lowest three adiabatic excited states of methylene with progressively larger JastrowSlater multideterminant complete active space CAS wave functions [3]. The highest of these states has the same symmetry, ${ }^{1} A_{1}$, as the first excited state. The DMC excitation energies obtained using any of the CAS wave functions are in excellent agreement with experiment. In contrast, single-determinant wave functions do not yield accurate DMC energies, indicating that it is important to include in the wave function Slater determinants that describe static strong correlation.

One of the reasons that quantum dots and quantum wires are interesting is that the system parameters can be tuned to create a very highly correlated system. In collaboration with the group of Harold Baranger at Duke we studied the localization of electrons in 
quantum dots and quantum wires $[4,5]$. Coulomb blockade phenomena are a useful probe of the crossover to strong correlation in quantum dots. Through calculations at low density using variational and diffusion quantum Monte Carlo up to $r_{s} \sim 55$, we found that the addition energy shows a clear progression from features associated with shell structure to those caused by a Wigner crystal. This crossover, which occurs near $r_{s} \sim 20$ for spin-polarized electrons, is a signature of interaction-driven localization. As the addition energy is directly measurable in Coulomb blockade conductance experiments, this provides a direct probe of localization in the low density electron gas. We also studied interaction-induced localization of electrons in an inhomogeneous quasi-one-dimensional system - a wire with two regions, one at low density and the other high. The phenomena observed depend on the density gradient - if it is steep, a barrier develops between the two regions, causing Coulomb blockade effects. The electrons in the low density region Wigner crystallize, whereas Friedel oscillations are observed at the edges of the highdensity regions. Ferromagnetic spin polarization does not appear for any parameters studied.

We developed a class of numerically efficient many-body wave functions to describe strongly correlated wave functions in any dimension called correlator product states [6]. Correlator product states introduce direct correlations between physical degrees of freedom in a simple way, yet provide the flexibility to describe a wide variety of systems. We showed that many interesting wave functions can be mapped exactly onto correlator product states, including Laughlins quantum Hall wave function, Kitaevs toric code states, and Huse and Elsers frustrated spin states. We also outlined the relationship between correlator product states and other common families of variational wave functions such as matrix product states, tensor product states, and resonating valence-bond states. Variational calculations for the Heisenberg and spinless Hubbard models demonstrated the promise of correlator product states for describing both two-dimensional and fermion correlations.

\section{References}

[1] J. Toulouse and C. J. Umrigar, J. Chem. Phys. 128, 174101 (2008)

[2] W. A. Al-Saidi and C. J. Umrigar, J. Chem. Phys. 128, 154324 (2008)

[3] P. M. Zimmerman, J. Toulouse, Z. Zhang, C. B. Musgrave, and C. J. Umrigar, J. Chem. Phys. 131, 124103 (2009)

[4] A. D. Güçlü, A. Ghosal, C. J. Umrigar, and H. U. Baranger, Phys. Rev. B 77, 041301 (2008)

[5] A. D. Güçlü, C. J. Umrigar, H. Jiang, and H. U. Baranger, Phys. Rev. B 80, 201302 (2009)

[6] H. J. Changlani, J. M. Kinder, C. J. Umrigar, and G. K.-L. Chan, Phys. Rev. B 80, 245116 (2009) 